\title{
In Vitro-In Vivo Correlation (IVIVC): From Current Achievements Towards the Future
}

\author{
Paweł Konrad Tuszyński ${ }^{1,}{ }^{*}$, Jakub Szlęk ${ }^{1}$, Sebastian Polak ${ }^{2,3}$, Renata Jachowicz ${ }^{1}$, and Aleksander \\ Mendyk ${ }^{1}$ \\ ${ }^{1}$ Department of Pharmaceutical Technology and Biopharmaceutics, Jagiellonian University Medical College, \\ Kraków, Poland \\ ${ }^{2}$ Department of Social Pharmacy, Unit of Pharmacoepidemiology and Pharmacoeconomics, Jagiellonian \\ University Medical College, Kraków, Poland \\ ${ }^{3}$ Certara UK, Sheffield, UK \\ e-mail: tuszek5@gmail.com
}

\section{ABSTRACT}

Conventional in vitro-in vivo correlation (IVIVC) based on compendial dissolution testing faces many obstacles, among which are problems in establishing meaningful correlation for immediate release dosage forms, lack of intravenous data in cases of many drugs without a possibility to obtain a unit impulse response, and well-known difficulties to build an IVIVC model for BCS III and BCS IV class drugs. Three major elements are obligatory for IVIVC development: in vitro dissolution data, in vivo pharmacokinetic profile, and modeling tools. Dissolution testing and modeling approaches are under heavy development to create predictive IVIVIC/IVIVR models. Application of noncompendial dissolution methods, biorelevant media, and equipment simulating the human gastrointestinal tract, together with sophisticated multivariate statistical methods and mechanistic approaches, are nominated to be the future of IVIVC.

KEYWORDS: Dissolution, IVIVC, IVIVR, biorelevant media, noncompendial dissolution tests, mechanistic modeling

\section{INTRODUCTION}

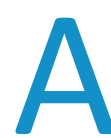
ccording to the US Federal Drug Administration's (FDA) guidance for industry, in vitro-in vivo correlation (IVIVC) is "the ability to predict, accurately and precisely, expected bioavailability characteristics for an [extended release (ER)] product from dissolution profile characteristics" (1). The established IVIVC reduces need for in vivo studies, which shortens drug development time and lowers overall cost. It also lowers the cost of post-approval changes, because once an IVIVC model is established, then a low-cost dissolution test could act as a surrogate for an expensive bioequivalence test. Moreover, a properly validated IVIVC model allows setting product specifications with dissolution acceptance criteria directly linked to the relevant plasma concentrations. Such applications of IVIVC have become a standard toolset in the modern drug development strategies employed by the pharmaceutical industry.

It is considered that the physicochemical properties of an active pharmaceutical ingredient (API) and the dosage form of the drug product are the main characteristics that limit the possibility of achieving IVIVC (2). In case of oral dosage forms, as highlighted by the FDA, it is always possible to correlate in vitro and in vivo data for formulations where the absorption of the API is limited by the dissolution rate. Therefore, it would be easiest to achieve IVIVC for compounds of BCS class II (BCS class III in some cases) and the ER formulations. In some cases, it is possible to establish IVIVC for APIs of other BCS classes and dosage forms (e.g., immediate release, inhalers, or transdermal). In addition to the API and dosage form characteristics, there are three main points that have to be considered during development of IVIVC: in vitro and in vivo study designs and modeling approach (2).

\section{Levels and Methods of Correlation}

The FDA recognizes four levels of IVIVC: Level A, B, C, and multiple Level $C$. The most desired is the Level A category of IVIVC. It is defined as a point-to-point relationship between in vitro dissolution and the in vivo response, such as plasma drug concentration or amount of drug absorbed.

According to the Guidance, there are two methods of establishing IVIVC, one-stage and two-stage. On one hand, the one-stage convolution approach uses the in vitro dissolution data and pharmacokinetic characteristics of the drug to obtain plasma drug concentration. On the other hand, the most common form of developing Level A correlation is the two-stage procedure, which involves development of formulations with different release rates

${ }^{*}$ Corresponding author. 
and their dissolution testing, providing in vivo plasma concentrations for the formulations, finding in vivo absorption or dissolution time profile using deconvolution (1). The deconvolution can be performed using either model-dependent or model-independent methods. The prior methods are constrained by the pharmacokinetic model, either one compartment (Wagner-Nelson) or two compartment (Loo-Riegelman) $(3,4)$. The latter is a more general approach, yet less stable than the former (1). According to survey conducted by Fotaki et al., the majority of IVIVC types of modeling approaches are using deconvolution and the simple linear models to achieve Level-A correlation (71\%) (5). Moreover, as Suarez-Sharp et al. pointed out, most new drug applications (NDAs) use a two-step model-independent approach to establish IVIVC (6). During recent years, a strong increase of scientific publications with the focus on IVIVC has been observed.

\section{Deconvolution}

Numerous case studies have reported the usage of Wagner-Nelson or Loo-Riegelman approaches, therefore academia seems to follow the trend set by industry and regulators. Cheng et al. for example, in two separate studies investigated the ionic-driven osmotic pump tablets consisting of theophylline or propranolol as model drugs $(7,8)$. The IVIVCs were established in the two-stage modeling by the Wager-Nelson method between an in vitro dissolution test and in vivo plasma drug concentrations obtained in an animal study. Moreover, Zhang et al. focused on matrix sustained-release tablets consisting of venlafaxine hydrochloride, chitosane, and carbomer (9). They developed a Level A IVIVC by the Wagner-Nelson method. In another study, Shin et al. compared the data from in vitro permeation tests and clinical studies for nicotine transdermal delivery systems (10). The analysis produced satisfactory results with a maximum prediction error less than $11 \%$ for the peak plasma concentration $\left(C_{\max }\right)$ and less than $8 \%$ for area under the curve (AUC) parameters. The IVIVC (polynomial linear model) was also developed with the two-stage Wagner-Nelson method. Other studies also indicate that in many cases the WagnerNelson equation is used for obtaining the absorption data, which is then plotted against dissolution data and processed in a linear regression, logistic, or polynomial manner (11-18). Next, the satisfactory results in terms of a correlation coefficient and convoluted in vivo data are presented; although, in most cases no external validation is done.

A more complex approach to establish IVIVC was done by Stillhart et al. and Ali et al. $(19,20)$. They used mechanistic absorption models to deconvolute in vivo data and mechanistic dissolution models to fit the in vivo dissolution profiles. The application of mechanistic models is elaborated more thoroughly in the section, "Mechanistic IVIVC".

Nevertheless, O'Hara et al. and Gillespie criticized the two-step approach, pointing out that the deconvolution step is unstable, and the predictions refer to the fraction of a dosage unit absorbed or dissolved rather than the primary focus, which is the plasma drug concentration $(21,22)$. The instability of deconvolution reflects not the particular method selection, but rather the influence of small changes of derivatives on the solutions to integral equations. The one-step approach advantages include direct modeling (in one step) of the relationship between measured quantities (in vitro dissolution and in vivo plasma drug concentrations), direct predictions of the plasma drug profile, and easier construction of methods that do not require a reference dose (22).

\section{Computational Intelligence Comes to Play}

There are new emerging methods that could overcome the above-mentioned obstacles. Various statistical computational intelligence tools have been applied to build IVIVC/IVIVR models. For example, Dunne et al. used a generalized linear mixed effects model (GLMM) (23). In general, the concept was to consider the time at which API dissolves/absorbs as a random variable. Then, the relating distribution functions were developed using proportional odds, proportional hazards, and proportional reversed hazards models. Furthermore, the parameters that relate in vivo and in vitro were allowed to change in time. Similar work was done by Kakhi et al., who treated the absorption process as a mixed effect (24). The derived system of equations was solved as part of a stochastic process that used a nonlinear mixed effects (NLME) engine. Moreover, Kortejärvi et al. and Qui et al. used the Bayesian likelihood function in a one-compartment model $(25,26)$. The IVIVC model consisted of prior knowledge and likelihood function, which defined the connection of the model parameters and the observed data.

The limitations of applying the classical IVIVC methods force researchers to seek more robust and general methods. Therefore, developing alternative methods for in vitro versus invivo data can be valuable, and it is expected that they will eventually be used beyond academia. To differentiate the classical IVIVC methodology from other methods, it is more suitable to use the term, "in vitro-in vivo relationship" (IVIVR), as it was discussed by Polli (27). Moreover, if the IVIVR transposes experimental results 
obtained in vitro to predict response-inducing properties, it becomes "in vitro-in vivo extrapolation" (IVIVE) (28). The first approach was made by Dowell et al., who used three types of artificial neural networks (feed forward, recurrent, jump connections, and general regression neural networks) to directly map the dissolution profile with pharmacokinetic observations (29). The resulting, so called, "input-output" associations were then externally assessed by calculating the correlation coefficient $\left(R^{2}\right)$, mean prediction error, and mean absolute error. Good results on the training and validation data $\left(R^{2}>0.85\right.$ and $>0.77$, respectively) have demonstrated the feasibility of this method in IVIVC/IVIVR procedures. Apart from that, there were few examples, where artificial neural networks (ANNs) were used to develop a relatively high level of IVIVC/IVIVR for sustained release dosage forms consisting of nifedipine or paracetamol (30-31). Later, the concept of using ANNs to correlate in vitro and in vivo data was extended by Mendyk et al., who included quantitative and qualitative composition of dosage formulations as covariates for in vitro data (32). Another example is a use of genetic programming (GP) in developing a relationship between in vitro and in vivo data. In the study by Yamashita et al., gene expression programming was used for optimizing in vitro-in vivo conversion function parameters (33). The new function was then used to calculate the in vivo absorption rate. $\mathrm{A}$ more general assumption was presented by Mendyk et al., who developed a direct relationship between in vitro dissolution data (input) and in vivo drug concentration (output) using GP, ANNs, and random decision forest models (34). Nevertheless, both groups emphasized that when using GP for oral dosage forms, the IVIVC provides relatively simple white-box models without the need to use intravenous injection data $(33,34)$.

\section{BIORELEVANT CONDITIONS}

\section{Standard Toolset}

Compendial dissolution testing methods have a wellestablished history of usage, mainly for quality control. The paddle (USP type 2 apparatus) and basket (USP type 1 apparatus) were the first dissolution equipment described and recommended by USP, and in principle, those are still used as testers for all oral dosage forms, both modified and immediate release. However, in terms of bioperformance prediction, the paddle and basket apparatuses have limitations related to less biorelevance because media composition changes over time. The media composition switch could be made only once, which is usually used to mimic dosage form transfer from stomach to intestines by simply alkalizing the media. Although for quality control it could be enough, for IVIVC a more complex system should be used to reflect the changes in physiology as the drug passes through the gastrointestinal (GI) tract. Successful IVIVC using the basket apparatus was established for metoprolol ER, metoprolol immediate release (IR), ibuprofen ER capsules, and novel once-daily ketoprofen ER formulations (35-38). Examples of drugs with IVIVC developed based on the paddle apparatus are griseofulvin fast disintegrating tablets, diltiazem MR, alprazolam controlled-release tablet, carbamazepine IR, diclofenac osmotic pump tablets, and montelucast $I R$ tablets (39-44).

The reciprocating cylinder (USP type 3 apparatus) is another compendial method, but not as widely used as paddle or basket due to inability to obtain sink conditions in many cases. The type 3 apparatus, however, offers a possibility to move a dosage form into different media over time, which reflects the GI fluid composition changes. If combined with biorelevant media, the type 3 apparatus could be an option to differentiate modified release dosage forms with variant dissolution rates, which is crucial step for establishing a meaningful IVIVC. Klein and Dressman used the type 3 apparatus to simulate passage of metoprolol ER tablets through the GI tract and to discriminate the drug release behavior (45). USP apparatus 3 was later applied by Klein et al. to develop a physiologically relevant IVIVC for mesalazine (although caffeine was used as model drug to facilitate the measurements) (46).

The flow-through cell (USP type 4 apparatus) has been used for development of IVIVC for several drugs. Successful IVIVC models were built for montelucast tablets and danazol capsules, both poorly soluble drugs (47-49). The type 4 apparatus is a compendial solution in such cases, when sink conditions are hard to achieve, but also offers a possibility for dynamic changes of medium, which reflects corresponding physiological $\mathrm{pH}$ and $\mathrm{Gl}$ fluid composition transitions.

Biorelevant media can be used along with compendial dissolution and dynamic dissolution systems. Examples include simulated gastric fluid (SGF), simulated intestinal fluid (SIF), fasted state simulated gastric fluid (FaSSGF), fasted state simulated intestinal fluid (FaSSIF), fed state simulated intestinal fluid (FeSSIF), and its modifications (without pepsin, pancreatin, or bile compounds). Simulating fluids are now widely available and used 
for quality control, new drug dosage formulation development, and IVIVC (50). Andreas et al. managed to predict dosage form-dependent food effects on extendedrelease nifedipine using simulated GI fluids (51). Wei et al. established the IVIVC of glyburide, a BCS class II drug, using FaSSIF and SIFF with a Caco-2 cell line to study drug permeability (52). As studies have shown, environmental changes have a big impact on solubilization of glyburide.

\section{Dynamic Dissolution}

Dynamic dissolution models have even more advantages. Dissolution test results are based on human conditions and less interpretations and computations are needed. The most notable examples of artificial GI tracts are the artificial stomach duodenum model (ADS), TNO TIM - 1, Golem apparatus, and stress-test devices developed by Garbacz et al. (53-58). Although those systems are expected to increase the likelihood of successful IVIVC when compendial methods fail, further research is needed to achieve more satisfying correlations in terms of bioperformance prediction.

The ADS model was used primarily to evaluate the effect of gastric emptying rate on API dissolution and solubilization (53). It consists only of a stomach chamber and duodenum chamber, transfer pumps, and fluid pumps. This system was used to predict carbamazepine solubilization in fasted and fed states in dogs. Another example of a Gl system is a biorelevant dissolution stresstest device built by Garbacz et al. (57). The stress-test device can mimic irregular movements and the velocity of a dosage form, along with segmented fluid distribution. Therefore the device is able to evaluate ER dosage forms in terms of integrity during dissolution (58). However, further work is needed to make it useful for biorelevant IVIVC model development.

Another artificial GI tract is known as TNO TIM-1, which was primarily used to simulate digestive and physiological processes in the human stomach and small intestine. TIM-1 consists of stomach, duodenum, jejunum, and ileum chambers and pumps. Acetaminophen was the first drug to be tested in this device, and now several other compounds were used to evaluate its ability to properly predict drug bioperformance, namely paroxetine IR tablets and mesalazine colonic delivery system (59-61).

The last notable example of dynamic dissolution systems is the Golem apparatus, a computer-controlled artificial digestive tract designed specifically for dissolution testing of oral dosage forms $(55,62)$. Like TIM-1, Golem also consists of four compartments and is a simplified version that can be operated by single person, and all complex functions are based on simple technical solutions. Instruments allow users to test a dosage form with different media including liquid and semi-solid food. Čulen et al. described two methods of utilizing Golem to establish IVIVC/IVIVR model for atorvastatin IR tablets (63).

\section{MECHANISTIC IVIVC}

\section{What it is and How it can be Used}

All of the above mentioned conventional methods cover most of the routineanalyses and commonly met situations; however, as industry interest goes beyond BCS class I drugs, more complex models and flexible approaches are needed. Complex absorption, distribution, metabolism, and excretion (ADME) processes regulating drug behavior create a need for separate estimation of various processes that are involved in the systemic absorption of drugs (dissolution, GI transit time, permeation, gut wall metabolism, and first pass metabolism). In such situation simple pharmacokinetic models, disregarding complex physiological phenomena is not sufficient. The suggested solution would be to develop and utilize a mechanistic IVIVC. In principle, the general methodology remains the same for the numerical or compartmental (both empirical) deconvolution, which aim in estimating the rate of drug input into the systemic circulation. Due to its character, the approach based on the mechanistic models can separate systemic input from in vivo dissolution. Therefore, in vivo dissolution rather than input rate can be directly correlated with its in vitro counterpart.

The approach is based on the physiologically based pharmacokinetic (PBPK) models, which describe mechanistically the absorption process. With their use, it is possible to integrate the drug or formulation, system data, and trial design-specific information with the retained possibility of separate analysis of each component. Compound and formulation data come from in vitro assays performed during the process of drug discovery and development. System data include human-specific anatomical and physiological parameters influencing drug disposition at the level of the GI tract and the whole body.

\section{Regulatory View and Industrial Awareness of the Approach}

As expected, the traditional IVIVC techniques are widely accepted and highly regulated and are useful tools for the pharmaceutical industry scientists. There is increasing awareness and utilization of the PBPK modelbased mechanistic IVIVC (64). Such techniques, although 
novel and requiring active research towards continuing advancement, remain a critical core technology area for the generic drug review function at the FDA (65).

\section{Case Studies}

Mistry et al. analyzed the previously published data generated in normal healthy volunteers taking metoprolol (66). A mechanistic modeling approach was applied to establish an IVIVC with the use of an Advanced Dissolution, Absorption and Metabolism (ADAM) model implemented in a Simcyp simulator. Authors tested multiple scenarios and discussed the impact of various parameters on the established IVIVC, including physiological parameters (gastric emptying time) and population variability in general. Grbic and colleagues used an Advanced Compartmental and Transit (ACAT) model implemented in GastroPlus to develop a drug-specific absorption model for gliclazide (GLK) (67). The generated absorption model provided the target in vivo dissolution profile for IVIVC and identification of biorelevant dissolution specifications for GLK IR tablets.

\section{SUMMARY AND OUTLOOK}

Although complex systems are being developed and regulators allow nonconventional modeling techniques to be used, provided they meet specified criteria for predictability, conventional IVIVC is mainly established and presented in $\operatorname{NDAs}(6,68)$. All the newer methods for achieving IVIVC are still in early development phases and are too new to be routinely used for regulatory purposes. Dynamic dissolution tests must be further developed and evaluated to reflect human tract conditions more reliably. Most artificial intelligence methods today are heuristic and difficult to validate completely. Mechanistic modeling is complicated and complex at the same time; therefore, to be available for all cases requires a lot of development, conceptually and technically. We believe that the state of the matters outlined in this paper indicates a bright future for IVVIC/IVIVR to be achieved by simultaneous development of dissolution and modeling methodologies.

\section{ACKNOWLEDGEMENTS}

The authors disclosed no funding related to this article.

\section{CONFLICT OF INTEREST}

The authors disclosed no conflicts of interest related to this article.

\section{REFERENCES}

1. Extended Release Oral Dosage Forms: Development, Evaluation, and Application of In Vitro/In Vivo Correlations; Guidance for
Industry; U.S. Department of Health and Human Services, Food and Drug Administration, Center for Drug Evaluation and Research (CDER), U.S. Government Printing Office: Washington, DC, 1997.

2. Cardot, J.-M.; Beyssac, E.; Alric, M. In vitro-in vivo correlation: importance of dissolution in ivivc. Dissolution Technol. 2007, 14, 15-19. DOI: 10.14227/dt140107p15.

3. Wagner, J. G.; Nelson, E. Per cent absorbed time plots derived from blood level and/or urinary excretion data. J. Pharm. Sci. 1963, 52, 610-611. DOI: 10.1002/jps.2600520629.

4. Loo, J. C. K.; Riegelman, S. New method for calculating the intrinsic absorption rate of drugs. J. Pharm. Sci. 1968, 57, 918928.

5. Fotaki, N.; Gray, V.; Kesisoglou, F.; Mayock, S.; Mirza, T.; Salt, A.; Selen, A. Survey results for in vitro-in vivo correlations (IVIVC): critical variables for success. Dissolution Technol. 2013, 20, 4850. DOI: $10.14227 / \mathrm{dt200213p48.}$

6. Suarez-Sharp, S.; Li, M.; Duan, J.; Shah, H.; Seo, P. Regulatory experience with in vivo in vitro correlations (IVIVC) in new drug applications. AAPS J. 2016, 18, 1379-1390. DOI: 10.1208/ s12248-016-9966-2.

7. Cheng, L.; Gao, S.; Ouyang, D.; Wang, H.; Wang, Y.; Pan, W.; Yang, $X$. Release mechanism between ion osmotic pressure and drug release in ionic-driven osmotic pump tablets (I). AAPS Pharmscitech. 2018, 19, 803-811. DOI: 10.1208/s12249-0170900-4.

8. Cheng, L.; Gai, X.; Wen, H.; Liu, D.; Tang, X.; Wang, Y.; Wang, T.; Pan, W.; Yang, $X$. Aqueous polymer dispersion coating used for osmotic pump tablets: membrane property investigation and ivivc evaluation. AAPS PharmSciTech 2018, 19, 242-250. DOI: 10.1208/s12249-017-0837-7.

9. Zhang, X.; Gu, X.; Wang, X.; Wang, H.; Mao, S. Tunable and sustained-release characteristics of venlafaxine hydrochloride from chitosan-carbomer matrix tablets based on in situ formed polyelectrolyte complex film coating. Asian J. Pharm. Sci. Published online: 2 Mar 2018. DOI: 10.1016/j.ajps.2018.01.004.

10. Shin, S. H.; Thomas, S.; Raney, S. G.; Ghosh, P.; Hammell, D. C.; El-Kamary, S. S.; Chen, W. H.; Billington, M. M.; Hassan, H. E.; Stinchcomb, A. L. In vitro-in vivo correlations for nicotine transdermal delivery systems evaluated by both in vitro skin permeation (IVPT) and in vivo serum pharmacokinetics under the influence of transient heat application. J. Control. Release 2018, 270, 76-88. DOI: 10.1016/j.jconrel.2017.11.034.ADDIN Mendeley Bibliography CSL_BIBLIOGRAPHYXMittapalli, R. K.; Marroum, P.; Qiu, Y.; Apfelbaum, K.; Xiong, H. Development of in vitro-in vivo correlation for potassium chloride extended release tablet formulation using urinary pharmacokinetic data. Pharm. Res. 2017, 34, 1527-1533. DOI: 10.1007/s11095-017-2179-2.

11. Mittapalli, R. K.; Nuthalapati, S.; Delke DeBord, A. E.; Xiong, H. Development of a level $A$ in vitro-in vivo correlation for veliparib (ABT-888) Extended release tablet formulation. Pharm. Res. 2017, 34, 1187-1192. DOI: 10.1007/s11095-017-2133-3. 
12. Andhariya, J. V.; Shen, J.; Choi, S.; Wang, Y.; Zou, Y.; Burgess, D. J. Development of in vitro-in vivo correlation of parenteral naltrexone loaded polymeric microspheres. J. Control. Release 2017, 255, 27-35. DOI: 10.1016/j.jconrel.2017.03.396.

13. Jirage, A.; Shaikh, K.; Vaishali, K.; Payghan, S. A.; Patwekar, S. In vitro-in vivo correlation for poly (3-hydroxybutyrate) base ibuprofen extended release tablets. Asian J. Pharm. 2017, 11, 18-26.

14. Xu, H.; Shi, Y.; Vela, S.; Marroum, P.; Gao, P. Developing quantitative in vitro -in vivo correlation for fenofibrate immediate-release formulations with the biphasic dissolutionpartition test method. J. Pharm. Sci. 2018, 107, 476-487. DOI: 10.1016/j.xphs.2017.06.018.

15. Narayanasamy, R.; Shabaraya, R. Development, internal and external validation of naproxen sodium sustained release formulation: a level A in vitro-in vivo correlation. Turkish J. Pharm. Sci. 2017, 14, 120-126. DOI: 10.4274/tjps.87587.

16. Honório Tda, S.; Pinto, E. C.; Rocha, H. V..; Esteves, V. S.; dos Santos, T. C.; Castro, H. C..; Rodrigues, C. R.; de Sousa, V. P.; Cabral, L. M. In vitro-in vivo correlation of efavirenz tablets using GastroPlus ${ }^{\circledR}$. AAPS PharmSciTech 2013, 14, 1244-1254. DOI: 10.1208/s12249-013-0016-4.

17. Cai, Y.; Li, Y.; Li, S.; Gao, T.; Zhang, L.; Yang, Z.; Fan, Z.; Bai, C. Level $A$ in vitro - in vivo correlation development and validation for tramadol hydrochloride formulations. Acta Pol. Pharm. 2016, 73, 1333-1338.

18. Stillhart, C.; Parrott, N. J.; Lindenberg, M.; Chalus, P.; Bentley, D.; Szepes, A. Characterising drug release from immediate-release formulations of a poorly soluble compound, basmisanil, through absorption modelling and dissolution testing. AAPS J. 2017, 19, 827-836. DOI: 10.1208/s12248-017-0060-1.

19. Ali, H.; Prasad Verma, P. R.; Dubey, S. K.; Venkatesan, J.; Seo, Y.; Kim, S.-K.; Singh, S. K. In vitro-in vivo and pharmacokinetic evaluation of solid lipid nanoparticles of furosemide using Gastroplus $^{\mathrm{TM}}$. RSC Adv. 2017, 7, 33314-33326. DOI: 10.1039/ C7RA04038E.

20. O’Hara, T.; Hayes, S.; Davis, J.; Devane, J.; Smart, T.; Dunne, A. In vivo-in vitro correlation (IVIVC) modeling incorporating a convolution step. J. Pharmacokinet. Pharmacodyn. 2001, 28, 277-298.

21. Gillespie, W. R. Convolution-based approaches for in vivo-in vitro correlation modeling. In In vitro-in vivo correlations.Young, D.B.; Devane, J.G.; Butler, J., Eds. Springer International Publishing, 1997; pp 53-65.

22. Dunne, A.; O'Hara, T.; Devane, J. Approaches to IVIVR modelling and statistical analysis. Adv. Exp. Med. Biol. 1997, 423, 67-86.

23. Kakhi, M.;Suarez-Sharp, S.;Shepard, T.; Chittenden, J. Application of an NLME-Stochastic deconvolution approach to level A IVIVC modeling. J. Pharm. Sci. 2017, 106, 1905-1916. DOI: 10.1016/j. xphs.2017.03.015.

24. Kortejärvi, H.; Mikkola, J.; Bäckman, M.; Antila, S.; Marvola, M. Development of level $A, B$ and $C$ in vitro - in vivo correlations for modified-release levosimendan capsules. Int. J. Pharm. 2002, 241, 87-95. DOI: 10.1016/S0378-5173(02)00137-0.

25. Qiu, J.; Martinez, M.; Tiwari, R. Evaluating in vivo-in vitro correlation using a Bayesian approach. AAPS J. 2016, 18, 619634. DOI: 10.1208/s12248-016-9880-7.

26. Polli, J. E. IVIVR versus IVIVC. Dissolution Technol. 2000, 7, 6-16. DOI: 10.14227/DT070300P6.

27. Tuszyński, P. K.; Polak, S.; Jachowicz, R.; Mendyk, A. From in vitroin vivo relationship (IVIVR) towards in vitro-in vivo extrapolation (IVIVE): a case study of pulmonary delivery systems. Dissolution Technol. 2017, 24, 32-35. DOI: 10.14227/DT240417P32.

28. Dowell, J. A.; Hussain, A.; Devane, J.; Young, D. Artificial neural networks applied to the in vitro-in vivo correlation of an extended-release formulation: initial trials and experience. $J$. Pharm. Sci. 1999, 88, 154-160. DOI: 10.1021/js970148p.

29. Ilić, M.; Đuriš, J.; Kovačević, I.; Ibrić, S.; Parojčić, J. In vitro - in silico - in vivo drug absorption model development based on mechanistic gastrointestinal simulation and artificial neural networks: nifedipine osmotic release tablets case study. Eur. J. Pharm. Sci. 2014, 62, 212-218. DOI: 10.1016/j.ejps.2014.05.030.

30. Parojčić, J.; Ibrić, S.; Djurić, Z.; Jovanović, M.; Corrigan, O. I. An investigation into the usefulness of generalized regression neural network analysis in the development of level $A$ in vitroin vivo correlation. Eur. J. Pharm. Sci. 2007, 30, 264-272. DOI: 10.1016/j.ejps.2006.11.010.

31. Mendyk, A.; Tuszyński, P.; Polak, S.; Jachowicz, R. Generalized in vitro-in vivo relationship (IVIVR) model based on artificial neural networks. Drug. Des. Dev. Ther. 2013, 7, 223-232. DOI: 10.2147/ DDDT.S41401.

32. Yamashita, F.; Fujita, A.; Zhang, X.; Sasa, Y.; Mihara, K.; Hashida, M. Computer-based evolutionary search for a nonlinear conversion function for establishing in vitro-in vivo correlation (IVIVC) of oral drug formulations. Drug Metab. Pharmacokinet. 2012, 27, 280-285. DOI: 10.2133/dmpk.DMPK-11-RG-075.

33. Mendyk, A.; Tuszyński, P. K.; Khalid, M. H.; Jachowicz, R.; Polak, S. How-to: empirical IVIVR without intravenous data. Dissolution Technol. 2015, 22, 12-18. DOI: 10.14227/DT220215P12.

34. Eddington, N. D.; Rekhi, G. S.; Lesko, L. J.; Augsburger, L. L. Scaleup effects on dissolution and bioavailability of propranolol hydrochloride and metaprolol tartrate tablet formulations. AAPS PharmSciTech 2000, 1, E14. DOI: 10.1208/pt010214.

35. Rekhi, G. S.; Eddington, N. D.; Fossler, M. J.; Schwartz, P.; Lesko, L. J.; Augsburger, L. L. Evaluation of in vitro release rate and in vivo absorption characteristics of four metoprolol tartrate immediate-release tablet formulations. Pharm. Dev. Technol. 1997, 2, 11-24. DOI: 10.3109/10837459709022605.

36. Honkanen, O.; Laaksonen, P.; Marvola,J.;Eerikainen, S.;Tuominen, $\mathrm{R}$;; Marvola, M.. Bioavailability and in vitro oesophageal sticking tendency of hydroxypropyl methylcellulose capsule formulations and corresponding gelatine capsule formulations. Eur. J. Pharm. Sci. 2002, 15, 479-488. DOI: 10.1016/S0928-0987(02)00032-5. 
37. Roda, A.; Sabatini, L.; Mirasoli, M.; Baraldini, M.; Roda, E. Bioavailability of a new ketoprofen formulation for once-daily oral administration. Int. J. Pharm. 2002, 241, 165-172. DOI: 10.1016/S0378-5173(02)00230-2.

38. Ahmed, I. S.; Aboul-Einien, M. H. In vitro and in vivo evaluation of a fast-disintegrating lyophilized dry emulsion tablet containing griseofulvin. Eur. J. Pharm. Sci. 2007, 32, 58-68. DOI: 10.1016/j. ejps.2007.05.114.

39. Korhonen, O.; Kanerva, H.; Vidgren, M.; Urtti, A.; Ketolainen, J. Evaluation of novel starch acetate-diltiazem controlled release tablets in healthy human volunteers. J. Control. Release 2004, 95, 515-520. DOI: 10.1016/j.jconrel.2003.12.026.

40. Mahaguna, V.; Talbert, R. L.; Peters, J. I.; Adams, S.; Reynolds, T. D.; Lam, F. Y. W.; Williams, R. O. III. Influence of hydroxypropyl methylcellulose polymer on in vitro and in vivo performance of controlled release tablets containing alprazolam. Eur. J. Pharm. Biopharm. 2003, 56, 461-468. DOI: 10.1016/S09396411(03)00116-4.

41. Jung, H.; Milán, R. C.; Girard, M. E.; León, F.; Montoya, M. A. Bioequivalence study of carbamazepine tablets: in vitro/in vivo correlation. Int. J. Pharm. 1997, 152, 37-44. DOI: 10.1016/ S0378-5173(97)04910-7.

42. Rani, M.; Mishra, B. Comparative in vitro and in vivo evaluation of matrix, osmotic matrix, and osmotic pump tablets for controlled delivery of diclofenac sodium. AAPS PharmSciTecH 2004, 5, 153-159. DOI: 10.1208/pt050471.

43. Zaid, A. N.; Natour, S.; Qaddomi, A.; Ghoush, A. A. Formulation and in vitro and in vivo evaluation of film-coated montelukast sodium tablets using Opadry ${ }^{\circledR}$ yellow 20 A82938 on an industrial scale. Drug Des. Devel. Ther. 2013, 7, 83-91. DOI: 10.2147/ DDDT.S37369.

44. Klein, S.; Dressman, J. B. Comparison of drug release from metoprolol modified release dosage forms in single buffer versus a pH-gradient dissolution test. Dissolution Technol. 2006, 12, 6-12. DOI: 10.14227/DT130106P6.

45. Klein, S.; Rudolph, M. W.; Skalsky, B.; Petereit, H.-U.; Dressman, J. B. Use of the BioDis to generate a physiologically relevant IVIVC. J. Control. Release 2008, 130, 216-219. DOI: 10.1016/j. jconrel.2008.06.014.

46. Okumu, A.; DiMaso, M.; Löbenberg, R. Dynamic dissolution testing to establish in vitro/in vivo correlations for montelukast sodium, a poorly soluble drug. Pharm. Res. 2008, 25, 2778-2785. DOI: 10.1007/s11095-008-9642-z.

47. Sunesen, V. H.; Vedelsdal, R.; Kristensen, H. G.; Christrup, L.; Müllertz, A. Effect of liquid volume and food intake on the absolute bioavailability of danazol, a poorly soluble drug. Eur. J. Pharm. Sci. 2005, 24, 297-303. DOI: 10.1016/j.ejps.2004.11.005.

48. Sunesen, V. H.; Pedersen, B. L.; Kristensen, H. G.; Müllertz, A. In vivo in vitro correlations for a poorly soluble drug, danazol, using the flow-through dissolution method with biorelevant dissolution media. Eur. J. Pharm. Sci. 2005, 24, 305-313. DOI: 10.1016/j.ejps.2004.11.007.
49. Dressman, J. Evolution of dissolution media over the last twenty years. Dissolution Technol. 2014, 21, 6-10. DOI: 10.14227/ DT210314P6.

50. Andreas, C. J.; Tomaszewska, I.; Muenster, U.; van der Mey, D.; Mueck, W.; Dressman, J. B. Can dosage form-dependent food effects be predicted using biorelevant dissolution tests? Case example extended release nifedipine. Eur. J. Pharm. Biopharm. 2016, 105, 193-202. DOI: 10.1016/j.ejpb.2016.06.010.

51. Wei, H.; Löbenberg, R. Biorelevant dissolution media as a predictive tool for glyburide a class II drug. Eur. J. Pharm. Sci. 2006, 29, 45-52. DOI: 10.1016/j.ejps.2006.05.004.

52. Carino, S. R.; Sperry, D. C.; Hawley, M. Relative bioavailability estimation of carbamazepine crystal forms using an artificial stomach-duodenum model. J. Pharm. Sci. 2006, 95, 116-125. DOI: 10.1002/jps.20495.

53. Verwei, M.; Minekus, M.;Zeijdner, E.; Schilderink, R.; Havenaar, R. Evaluation of two dynamic in vitro models simulating fasted and fed state conditions in the upper gastrointestinal tract (TIM-1 and Tiny-TIM) for investigating the bioaccessibility of pharmaceutical compounds from oral dosage forms. Int. J. Pharm. 2016, 498, 178-186. DOI: 10.1016/j.jpharm.2015.11.048.

54. Stupák, I.; Pavloková, S.; Vysloužil, J.; Dohnal, J.; Čulen, M. Optimization of dissolution compartments in a biorelevant dissolution apparatus golem v2, supported by multivariate analysis. Molecules 2017, 22, 2042. DOI: 10.3390/ molecules22122042.

55. Garbacz, G.; Klein, S. Dissolution testing of oral modified-release dosage forms. J. Pharm. Pharmacol. 2012, 64, 944-968. DOI: 10.1111/j.2042-7158.2012.01477.x.

56. Garbacz, G.; Kandzi, A.; Koziolek, M.; Mazgalski, J.; Weitschies, W. Release characteristics of quetiapine fumarate extended release tablets under biorelevant stress test conditions. AAPS PharmSciTech. 2014, 15, 230-236. DOI: 10.1208/s12249-0130050-2.

57. Garbacz, G.; Cadé, D.; Benameur, H.; Weitschies, W. Bio-relevant dissolution testing of hard capsules prepared from different shell materials using the dynamic open flow through test apparatus. Eur. J. Pharm. Sci. 2014, 57, 264-272. DOI: 10.1016/j. ejps.2013.08.039.

58. Blanquet, S.; Zeijdner, E.; Beyssac, E.; Meunier, J.-P.; Denis, S.; Havenaar, R.; Alric, M. A dynamic artificial gastrointestinal system for studying the behavior of orally administered drug dosage forms under various physiological conditions. Pharm. Res. 2004, 21, 585-591. DOI: 10.1023/B:PHAM.0000022404.7 0478.4b.

59. Naylor, T. A.; Connolly, P. C.; Martini, L. G.; Elder, D. P.; Minekus, M.; Havenaar, R.; Zeijdner, E. Use of a gastro-intestinal model and Gastroplus ${ }^{\mathrm{TM}}$ for the prediction of in vivo performance. Ind. Pharmacy 2006, 12, 9-12.

60. Tenjarla S, Romasanta V, Zeijdner E, Villa R, M. L. Release of 5-aminosalicylate from an MMX mesalamine tablet during 
transit through a simulated gastrointestinal tract system. Adv. Ther. 2007, 24, 826-840. DOI: 10.1007/BF02849976.

61. Čulen, M.; Dohnal, J. Advances in dissolution instrumentation and their practical applications. Drug Dev. Ind. Pharm. 2013, 40, 1277-1182. DOI: 10.3109/03639045.2013.841184.

62. Čulen, M.; Tuszyński, P. K.; Polak, S.; Jachowicz, R.; Mendyk, A.; Dohnal, J. Development of in vitro - in vivo correlation/ relationship modeling approaches for immediate release formulations using compartmental dynamic dissolution data from "golem": a novel apparatus. Biomed Res. Int. 2015, 328628. DOI: 10.1155/2015/328628.

63. Nguyen, M. A.; Flanagan, T.; Brewster, M.; Kesisoglou, F.; Beato, S.; Biewenga, J.; Crison, J.; Holm, R.; Li, R.; Mannaert, E. A survey on IVIVC/IVIVR development in the pharmaceutical industry - past experience and current perspectives. Eur. J. Pharm. Sci. 2017, 102, 1-13. DOI: 10.1016/j.ejps.2017.02.029.
64. Zhang, X.; Duan, J.; Kesisoglou, F.; Novakovic, J.; Amidon, G. L.; Jamei, M.; Lukacova, V.; Eissing, T.; Tsakalozou, E.; Zhao, L. Mechanistic oral absorption modeling and simulation for formulation development and bioequivalence evaluation: report of an FDA public workshop. CPT Pharmacometrics Syst. Pharmacol. 2017, 6, 492-495. DOI: 10.1002/psp4.12204.

65. Mistry, B.; Patel, N.; Jamei, M.; Rostami-Hodjegan, A.; Martinez, $M$. N. Examining the use of a mechanistic model to generate an in vivo/in vitro correlation: journey through a thought process. AAPS J. 2016, 18, 1144-1158. DOI: 10.1208/s12248-016-9930-1.

66. Grbic, S.; Parojcic, J.; Ibric, S.; Djuric, Z. In vitro-in vivo correlation for gliclazide immediate-release tablets based on mechanistic absorption simulation. AAPS PharmSciTech 2011, 12, 165-171. DOI: 10.1208/s12249-010-9573-y .

67. Gray, V. Meeting Report: PQRI workshop on application of IVIVC in formulation development. Dissolution Technol. 2012, 19, 5659. DOI: 10.14227/DT190412P56.

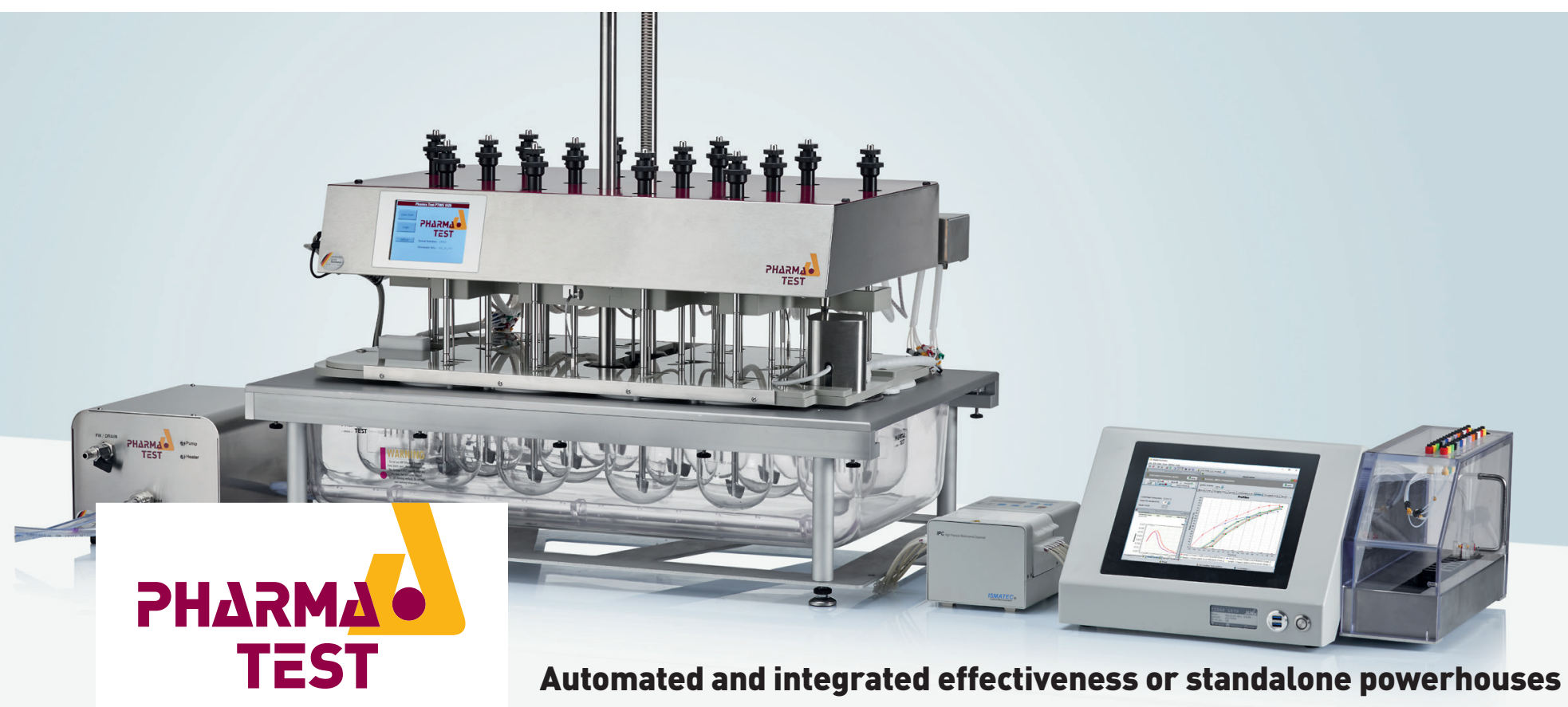

Powerful standalone solutions. Or a fully integrated automated system. It's „The German Gründlichkeit“.
The PTWS 1420 is a 14+2 position dissolution testing instrument. Ideal for comparative studies such as biowaiver tests. The PTWS 1420 is also available as the fully online automated tablet dissolution testing system ADS-L 1420 with automated sampling, measuring and result

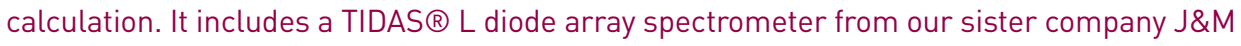
with an integrated PC running the fully 21 CFR part 11 compliant WinDiss ARGUS software. All instruments are fully USP/JP/CP/EP/USFDA/ASTM compliant. 